\title{
Long-term follow-up after bronchoscopic lung volume reduction in patients with emphysema
}

\author{
Federico Venuta, Marco Anile, Daniele Diso, Carolina Carillo, Tiziano De Giacomo, \\ Antonio D'Andrilli, Francesco Fraioli, Erino A. Rendina and Giorgio F. Coloni
}

ABSTRACT: Bronchoscopic lung volume reduction (BLVR) is a novel emphysema therapy. We evaluated long-term outcome in patients with heterogeneous emphysema undergoing BLVR with one-way valves.

40 patients undergoing unilateral BLVR entered our study. Pre-operative mean forced expiratory volume in $1 \mathrm{~s}(\mathrm{FEV} 1)$ was $0.88 \mathrm{~L} \cdot \mathrm{s}^{-1}(23 \%)$, total lung capacity was $7.45 \mathrm{~L}(121 \%)$, intrathoracic gas volume was $6 \mathrm{~L}(174 \%)$, residual volume (RV) was $5.2 \mathrm{~L}(232 \%)$, and the 6-min walk test (6MWT) was $286 \mathrm{~m}$. All patients required supplemental oxygen; the Medical Research Council (MRC) dyspnoea score was 3.9. High-resolution computed tomography (HRCT) results were reviewed to assess the presence of interlobar fissures.

33 patients had a follow-up of $>12$ months (median 32 months). $37.5 \%$ of the patients had visible interlobar fissures. $40 \%$ of the patients died during follow-up. Three patients were transplanted and one underwent lung volume reduction surgery. Supplemental oxygen, FEV1, RV, 6MWT and MRC score showed a statistically significant improvement $(p \leqslant 0.0001, p=0.004$, $p=0.03, p=0.003$ and $p<0.0001$, respectively). Patients with visible fissures had a functional advantage.

BLVR is feasible and safe. Long-term sustained improvements can be achieved. HRCT-visible interlobar fissures are a favourable prognostic factor.

KEYWORDS: Bronchoscopic lung volume reduction, emphysema, lung volume reduction surgery

mphysema is a worldwide leading cause of disability and death affecting $\sim 1.8 \%$ of the global population [1]. The current standard maximal medical treatment includes smoking cessation, administration of bronchodilators, pulmonary rehabilitation and long-term oxygen therapy; it allows improvement in exercise capacity and quality of life. However, it shows some limitations in the case of advanced disease. For this reason, according to radiological and functional details and the clinical status of the patients, a number of surgical procedures have been proposed historically, these include: bullectomy [2], single and double lung transplantation [3] and, more recently, lung volume reduction surgery (LVRS) [4]. The latter is based on the hypothesis that dyspnoea may be related to severe impairment of respiratory mechanics owing to increased end-expiratory lung volumes with lungs and thorax overinflation. In fact, when target areas of hyperinflated lungs are resected, the residual lung and chest wall mechanics are significantly improved with consequent symptomatic relief. However, LVRS gained popularity too fast and without control; this posed important questions regarding the real value of the procedure and the appropriate selection of patients. Patients with a more advanced functional deterioration had a higher mortality and less encouraging results, suggesting that LVRS should be considered more carefully in these situations [5].

Some authors have speculated that similar results could be achieved by less invasive bronchoscopic approaches, isolating and deflating the most hyperinflated parts of the emphysematous lungs. Several bronchoscopic alternatives have been proposed: endobronchial occluders [6], sealants [7], coils [8], steam [9] and airway by-pass [10, 11]. Bronchoscopic lung volume reduction (BLVR) with one-way valves has been attempted in the experimental laboratory [12] and in selected clinical settings [13-16]. A randomised clinical study [17] has contributed to validate feasibility, safety, and short- and medium-term effectiveness, allowing the procedure to advance one step further in the clinical arena.
AFFILIATIONS

*Dept of Thoracic Surgery, University of Rome Sapienza, Policlinico Umberto I, Fondazione Eleonora Lorillard Spencer Cenci, Rome, Italy.

CORRESPONDENCE

F. Venuta

Cattedra di Chirurgia Toracica,

Policlinico Umberto I

Università di Roma Sapienza

Fondazione Eleonora Lorillard

Spencer Cenci

Viale le del Policlinico 155

00161 Rome

Italy

E-mail: federico.venuta@uniroma1.it

Received:

April 282011

Accepted after revision:

Oct 012011

First published online:

Oct 172011 
We have previously reported a feasibility and safety study with short-term results [13]. We hereby report long-term results in a larger population of patients.

\section{PATIENTS AND METHODS}

We conducted a prospective, nonrandomised, single-centre longitudinal study to evaluate the long-term efficacy of BLVR performed by placing one-way endobronchial valves (Zephir ${ }_{\circledR}$ valves; Pulmonx Corp., Redwood City, CA, USA) in the bronchi supplying the most hyperinflated parts of the emphysematous lungs. 40 patients (37 males, three females; mean age $60.5 \pm 9.8$ yrs) were enrolled. The protocol was approved by the ethical committee of the Policlinico Umberto I (Sapienza University of Rome, Rome, Italy) (Prot. 350/03). Formal informed consent was obtained from each patient. Only patients undergoing unilateral BLVR were included; patients receiving bilateral treatment (included in our previous report) were excluded.

The inclusion and exclusion criteria are listed in table 1 . The critical point was the presence of marked hyperinflation with regional variations in the distribution of emphysema providing "target" areas ("surgical" heterogeneous disease). Thus, all patients had heterogeneous emphysema with one or more lobes clearly more compromised than the rest of the lung. Heterogeneity was subjectively assessed by at least two members of the team using high-resolution computed tomography (HRCT) and lung perfusion scan; we treated only lobes showing a clear density reduction with no perfusion. The presence of interlobar fissures was retrospectively blindly determined by our radiologist (F. Fraioli) using three-dimensional reconstructions at computed tomography and multiplanar evaluation. All patients received optimal medical therapy at the time of evaluation and all required supplemental oxygen. A formal rehabilitation programme was not required for this study, although all patients received long-term rehabilitation at the referral centre. The preoperative functional variables are reported in table 2.

On-site evaluation included physical examination, body plethysmography, diffusing capacity of the lung for carbon

\section{TABLE 1 Inclusion and exclusion criteria}

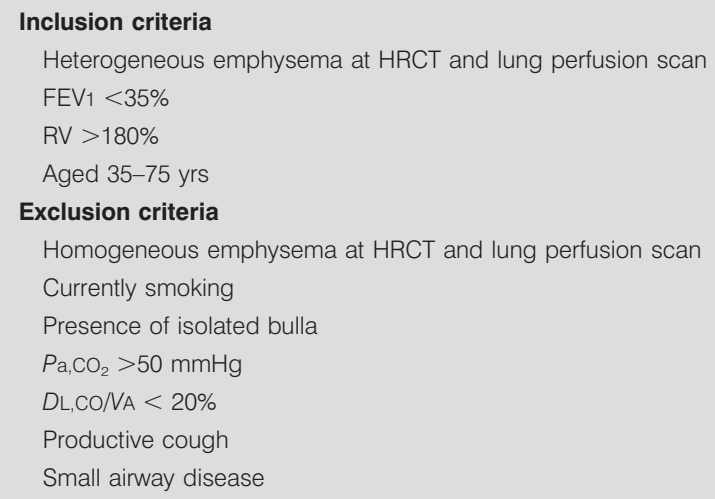

HRCT: high-resolution computed tomography; FEV1: forced expiratory volume in $1 \mathrm{~s}$; RV: residual volume; $\mathrm{Pa}_{\mathrm{a}, \mathrm{CO}_{2}}$ : arterial carbon dioxide tension; $\mathrm{DL}, \mathrm{CO} / \mathrm{VA}$ : diffusing capacity of the lung for carbon monoxide corrected for alveolar ventilation. monoxide corrected for alveolar ventilation ( $D \mathrm{~L}, \mathrm{CO} / V \mathrm{~A})$, arterial blood gas analysis, 6-min walk test (6MWT), chest radiograph, HRCT, lung perfusion scan and transthoracic echocardiography; dyspnoea was quantified by the Medical Research Council (MRC) grading system. Time-points for postoperative evaluation were after $24-72$ h, 1 and 3 months, and 1,3 and 5 yrs.

All the procedures were performed in the operating room under intravenous anaesthesia (propofol infusion) and with spontaneous assisted ventilation through an endotracheal tube or a laryngeal mask. Local anaesthesia (lydocaine $2 \%$ ) was administered within the target bronchus before deploying the valves to prevent coughing. The characteristics of the valves and the deployment technique have been previously reported by our group $[13,18]$.

Continuous variables are reported as mean $\pm \mathrm{SD}$. The variation of means during follow-up was compared by one-way ANOVA and post hoc tests (least significant difference, Bonferroni and Sidak) for multiple comparisons. The comparison during followup between means and either presence or absence of interlobar fissures was performed by unpaired t-tests. The correlation between the variables' trend and the presence or absence of interlobar fissures was calculated by the Spearman's rho correlation coefficient. All the statistical tests were two-tailed and a significance level of $p=0.05$ was accepted. Survival curves were calculated from valve placement to death or last follow-up and were constructed according to the Kaplan-Meier method. The log-rank test was performed to compare survival between patients with and without visible fissures. Statistical analysis was performed using SPSS 17.0 software for Windows (SPSS Inc, Chicago, IL, USA).

\section{TABLE 2 Pre-operative variables}

\begin{tabular}{|c|c|c|c|}
\hline Variable & Results & $\%$ pred & Range \\
\hline$F_{E V} \mathbf{L} \cdot \mathrm{s}^{-1}$ & $0.88 \pm 0.3$ & 23 & $15-51$ \\
\hline RV L & $5.2 \pm 0.9$ & 232 & $177-328$ \\
\hline TLC L & $7.45 \pm 1.1$ & 122 & $85-134$ \\
\hline FVC L & $2.0 \pm 0.9$ & 45 & $33-62$ \\
\hline ITGV L & $6.0 \pm 1.1$ & 174 & $134-220$ \\
\hline $\mathrm{Pa}, \mathrm{O}_{2} \mathrm{mmHg}$ & $72.7 \pm 11.3$ & & $57-102$ \\
\hline $\mathrm{Pa}, \mathrm{CO}_{2} \mathrm{mmHg}$ & $41.2 \pm 4.5$ & & $28-46$ \\
\hline $\mathrm{O}_{2}$ saturation $\%$ & $94.9 \pm 3.1$ & & $91.2-97.1$ \\
\hline$D L, C O / V A ~ m L \cdot m i n^{-1} \cdot \mathrm{mmHg}^{-1} \cdot \mathrm{L}^{-1}$ & $2.95 \pm 1.9$ & 33 & $27-76$ \\
\hline Supplemental $\mathrm{O}_{2} \mathrm{~L} \cdot \mathrm{min}^{-1}$ & $1.87 \pm 1.2$ & & $1-3$ \\
\hline 6-min walking test $\mathrm{m}$ & $286 \pm 72$ & & $124-458$ \\
\hline MRC scale & $3.9 \pm 0.8$ & & $3-5$ \\
\hline Visible fissures at HRCT $n$ & 15 & & \\
\hline
\end{tabular}

$\%$ pred: \% predicted; FEV 1 : forced expiratory volume in $1 \mathrm{~s}$; RV: residual volume; TLC: total lung capacity; FVC: forced vital capacity; ITGV: intrathoracic gas volume; $\mathrm{Pa}_{2} \mathrm{O}_{2}$ : arterial oxygen tension; $\mathrm{Pa}_{1} \mathrm{CO}_{2}$ : arterial carbon dioxide tension; $\mathrm{LL}, \mathrm{CO} / \mathrm{VA}$ : diffusing capacity of the lung for carbon monoxide corrected for alveolar ventilation; MRC: Medical Research Council; HRCT: high-resolution computed tomography. 


\section{RESULTS}

142 valves were placed with a mean of 3.6 per patient; 39 (27.5\%) valves were placed in the left upper lobe, $66(46.8 \%)$ in the right upper lobe, four $(2.8 \%)$ in the middle lobe, $20(14.1 \%)$ in the right lower lobe and 13 (9.3\%) in the left lower lobe. The devices were placed in the left upper lobe in 13 patients $(32.5 \%)$, in the right upper lobe in $17(42.5 \%)$, in the right upper and middle lobe in two $(5 \%)$, in the right lower lobe in five $(12.5 \%)$ and in the left lower lobe in three $(7.5 \%)$. Thus, the middle lobe was always treated together with the upper lobe. The median operative time was $39 \mathrm{~min}$ (range 15-95 $\mathrm{min}$ ). The median follow-up was 32 months. 33 patients were evaluated after 1 yr, 18 after 3 yrs and nine after 5 yrs.

No intra-operative complications were observed. The mean hospital stay was 5 days (range 2-32 days) One contralateral pneumothorax occurred 15 days after the procedure while the patient was at home. Two patients had pneumonia in the lobe adjacent to where the valves were inserted. One patient requiring anticoagulation after coronary artery revascularisation presented mild haemoptysis 3 yrs after valve placement; in this patient no endobronchial abnormalities related to the presence of the valves were endoscopically detected. Two patients had small granulations growing in front of the valve; however, they required no treatment, as the device was not obstructed and there were no symptoms. Two patients underwent single lung transplantation (SLT) and one received double lung transplantation (DLT) at a mean of 6.3 months after valve placement; two of them died after the transplant with no valve-related complications and one is still alive and well. The two SLT patients were transplanted on the side where valves were previously placed. One patient died of respiratory failure while on the waiting list for DLT 13 months after valve placement; in this patient, fibreoptic bronchoscopy was performed through the tracheostomy during the last hospitalisation and all the valves previously placed were patent. One patient is currently on the waiting list for transplantation. One patient underwent LVRS 1 yr after valve placement; one patient had the valves removed at another centre after 3 months; the latter was not included in the survival analysis. $16(40 \%)$ patients died during follow-up (lung cancer in four (25\%); myocardial infarction with intractable arrhythmia in three $(18.7 \%)$; end-stage respiratory failure in seven (43.8\%); and post-transplant in two $(12.5 \%))$. The functional results at each time-point are reported in table 3 . No significant modification was observed immediately after the procedure. Only two patients experienced complete lobe atelectasis 1 and 3 weeks after the procedure. At 1 and 3 months there was a significant improvement in terms of forced expiratory volume in $1 \mathrm{~s}$ (FEV1) and a decrease in the residual volume (RV); also total lung capacity (TLC) and intrathoracic gas volume decreased slightly without reaching statistical significance and the 6MWT improved significantly. Overall, most of the patients showed an improvement of the MRC score with a significant reduction of symptoms, persisting after 1, 3 and 5 yrs. Most of the patients required less supplemental oxygen with a stable mean arterial oxygen tension and oxygen saturation. Improvement in terms of oxygen requirement, FEV1, forced vital capacity (FVC), 6MWT and MRC score remained stable during follow-up. Post hoc tests confirmed that most of the improvement was during the first year. However, 6MWT and MRC improvements are significant at all time-points; supplemental oxygen is significant up to the third year, RV is significant at 1 and 5 yrs and FVC improvement is significant at $1 \mathrm{yr}$, but it is not significant for the complete duration of follow-up. The median pre-operative FEV1 was $0.77 \mathrm{~L} \cdot \mathrm{s}^{-1}$, patients were stratified according to this value into two groups (20 patients in each group); the group of patients below the median FEV1 showed a $28 \%$ FEV1 improvement (from a mean of 0.65 to $0.83 \mathrm{~L} \cdot \mathrm{s}^{-1}$ ) and the group above the median had a

TABLE 3 Functional variables of the patients at the different time-points

\begin{tabular}{|c|c|c|c|c|c|c|c|c|c|}
\hline \multirow[t]{2}{*}{ Variables } & \multirow[t]{2}{*}{ Baseline } & \multicolumn{7}{|c|}{ Follow-up } & \multirow[t]{2}{*}{$\mathrm{p}$-value } \\
\hline & & 24-72 h & 1 month & 3 months & 6 months & $1 \mathrm{yr}$ & 3 yrs & $5 \mathrm{yrs}$ & \\
\hline $\mathrm{O}_{2}$ saturation $\%$ & $94.9 \pm 3.1$ & $95.2 \pm 2.9$ & $94.8 \pm 2.9$ & $95.1 \pm 1.8$ & $94.9 \pm 1.9$ & $94.7 \pm 1.9$ & $94.4 \pm 1.9$ & $95.7 \pm \pm 2.4$ & 0.2 \\
\hline FEV $1 \mathrm{~L} \cdot \mathrm{min}^{-1}$ & $0.88 \pm 0.3$ & $0.9 \pm 0.3$ & $1.1 \pm 0.2$ & $1.1 \pm 0.3$ & $1.1 \pm 0.3$ & $1.09 \pm 0.4$ & $1.08 \pm 0.4$ & $1.2 \pm 0.5$ & 0.004 \\
\hline FVC L & $2.0 \pm 0.6$ & $2.1 \pm 0.5$ & $2.3 \pm 0.6$ & $2.3 \pm 0.8$ & $2.3 \pm 0.5$ & $2.4 \pm 0.6$ & $2.4 \pm 0.5$ & $2.5 \pm 0.6$ & 0.06 \\
\hline RV L & $5.2 \pm 0.9$ & $4.5 \pm 0.8$ & $4.7 \pm 1.0$ & $4.7 \pm 0.9$ & $4.4 \pm 1.1$ & $4.4 \pm 1.2$ & $4.4 \pm 1.2$ & $3.98 \pm 1.3$ & 0.03 \\
\hline TLC L & $7.45 \pm 1.1$ & $7.39 \pm 1.3$ & $7.49 \pm 1.1$ & $7.41 \pm 0.9$ & $7.3 \pm 1.1$ & $7.28 \pm 1.0$ & $7.29 \pm 1.1$ & $7.3 \pm 1.3$ & 0.7 \\
\hline ITGV L & $6.0 \pm 1.1$ & $5.1 \pm 0.9$ & $5.4 \pm 1.0$ & $5.4 \pm 1.1$ & $5.3 \pm 1.0$ & $5.3 \pm 1.1$ & $5.2 \pm 1.3$ & $5.3 \pm 1.2$ & 0.1 \\
\hline $\begin{array}{c}\text { DL,Co/VA mL } \cdot \min ^{-1} . \\
\mathrm{mmHg}^{-1} \cdot \mathrm{L}^{-1}\end{array}$ & $2.95 \pm 1.9$ & $3.03 \pm 1.9$ & $2.99 \pm 1.8$ & $2.81 \pm 1.5$ & $2.84 \pm 1.2$ & $2.88 \pm 1.5$ & $3.35 \pm 1.3$ & $3.86 \pm 1.2$ & 0.2 \\
\hline
\end{tabular}

Data are presented as mean $\pm \mathrm{SD}$, unless otherwise stated. $\mathrm{Pa}_{\mathrm{a}} \mathrm{O}_{2}$ : arterial oxygen tension; $\mathrm{Pa}_{1} \mathrm{CO}_{2}$ : arterial carbon dioxide tension; FEV1: forced expiratory volume in $1 \mathrm{~s}$; FVC: forced vital capacity; RV: residual volume; TLC: total lung capacity; ITGV: intrathoracic gas volume; DL,CONA: diffusing capacity of the lung for carbon monoxide corrected for alveolar ventilation; 6MWT: 6-min walking test; MRC: Medical Research Council. 


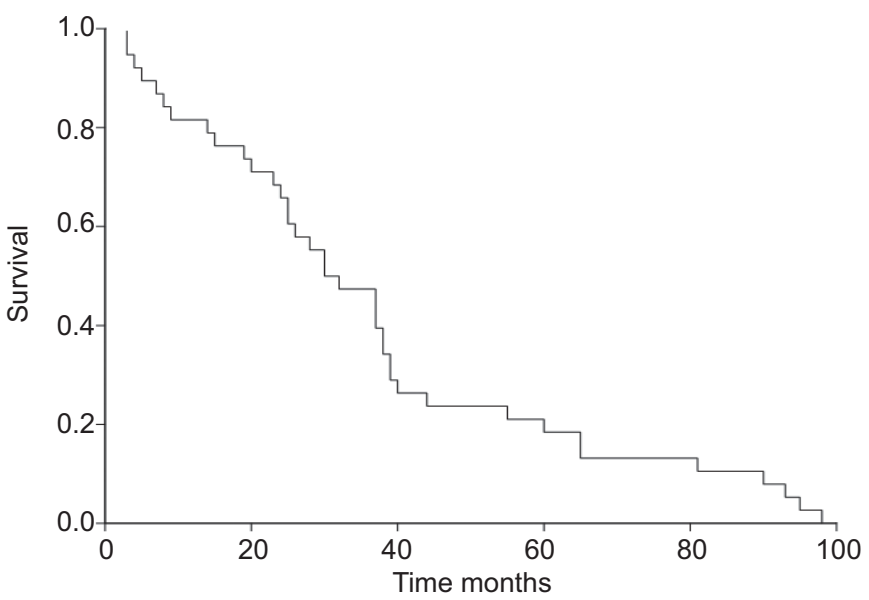

FIGURE 1. Survival according to the Kaplan-Meier method.

$12.6 \%$ improvement (from 1.11 to $1.25 \mathrm{~L} \cdot \mathrm{s}^{-1}$ ). Both improvements were statistically significant ( $p<0.0001$ and $p<0.03$, respectively). However, there were no statistically significant differences between the two groups. The same stratification was performed below and above the median RV without observing any statistically significant difference.

The mean and median survivals were $36 \pm 4.3$ and $30 \pm 4.6$ months, respectively. Survival at 1,3 and 5 yrs were $81.6 \%, 47.4 \%$ and $22.4 \%$, respectively. Actuarial survival is shown in figure 1 .

The review of HRCT allowed visualisation of the interlobar fissures in 15 (37.5\%) out of 40 patients. The relative percentage of patients with visible fissures increased during follow-up $(45.5,50$ and $72 \%$ at 1,3 and 5 yrs, respectively). Functional results with respect to presence/absence of interlobar fissures are reported in table 4, demonstrating some functional advantages for patients with visible fissures. Mortality during follow-up was higher in the group of patients without HRCT visible interlobar fissures, as confirmed by the log-rank test (fig. 2).

\section{DISCUSSION}

LVRS is the greatest advancement in chronic obstructive pulmonary disease (COPD) surgical management since the development of lung transplantation $>30$ yrs ago. It certainly provides a reliable palliation of symptoms in a well-selected group of patients. The National Emphysema Treatment Trial (NETT) trial reported improvements in survival and functional benefits in those with upper lobe predominant heterogeneous disease and limited exercise capacity [19]. However, this procedure still carries a relatively high price tag with poor costeffectiveness related to the number of adverse clinical outcomes, and the potentially prolonged hospitalisation and long-term care required [20]. Patients with the most advanced disease show a high mortality rate and achieve less favourable results, suggesting caution in case of excessively low FEV1 and either homogeneous disease or very low $D L, C O$ [5].

For these reasons, many investigators have pursued research into innovative and alternative methods to achieve similar results of reducing morbidity and costs. Both thoracic surgeons and pulmonologists have considered whether emphysema palliation might be accomplished endoscopically, possibly becoming an outpatient procedure. These new endoscopic procedures should also be seen as an opportunity to benefit a larger group of symptomatic patients who may not be candidates for LVRS or transplantation, or bridge them to these operations, allowing

TABLE 4 Functional variables at 1, 3 and 5 yrs compared according to the presence or absence of interlobar fissures visible at high-resolution computed tomography

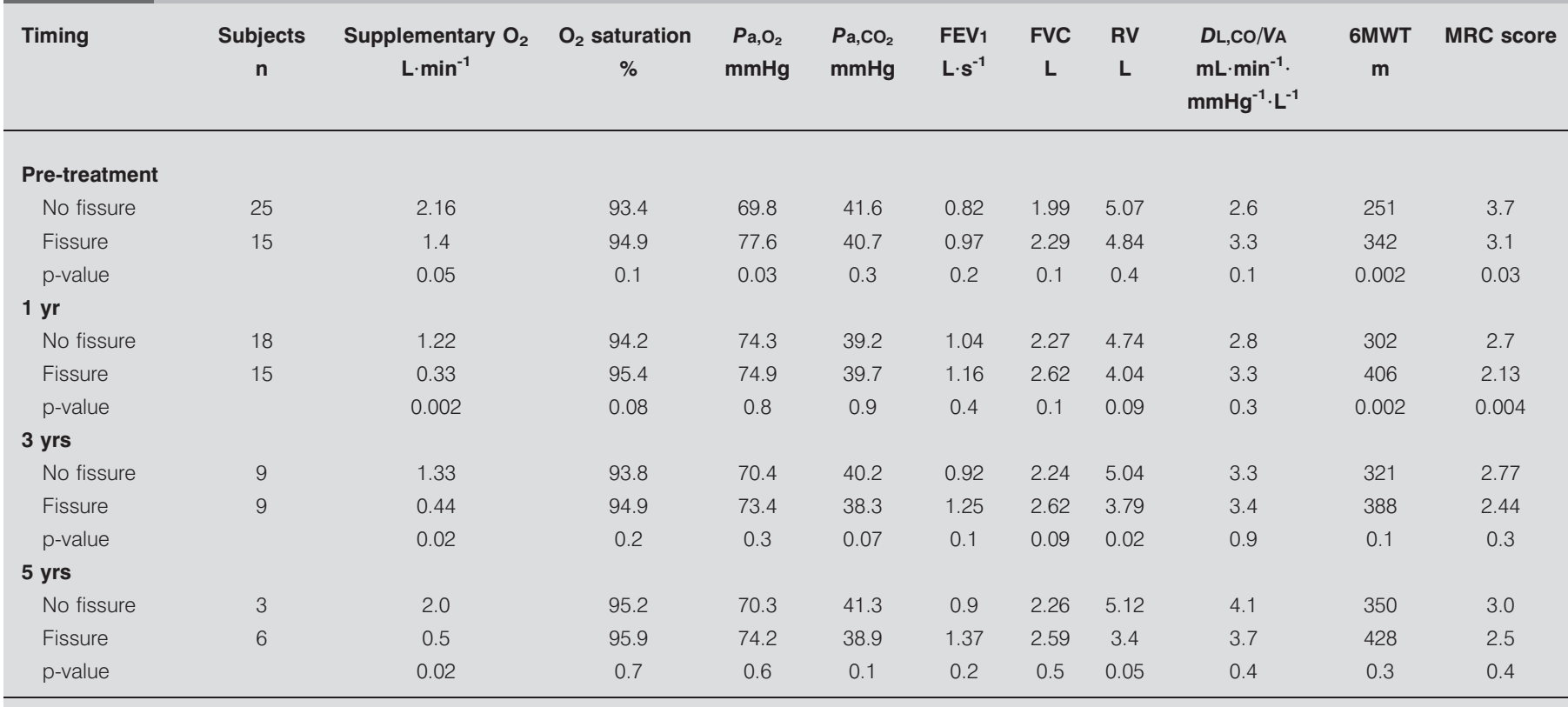

$\mathrm{Pa}_{\mathrm{a}} \mathrm{O}_{2}$ : arterial oxygen tension; $\mathrm{Pa}_{\mathrm{a}} \mathrm{CO}_{2}$ : arterial carbon dioxide tension; $\mathrm{FEV} 1$ : forced expiratory volume in $1 \mathrm{~s}$; $\mathrm{FVC}$ : forced vital capacity; $\mathrm{RV}$ : residual volume; $\mathrm{DL}, \mathrm{CO} / \mathrm{VA}$ diffusing capacity of the lung for carbon monoxide corrected for alveolar ventilation; 6MWT: 6-min walking test; MRC: Medical Research Council. 


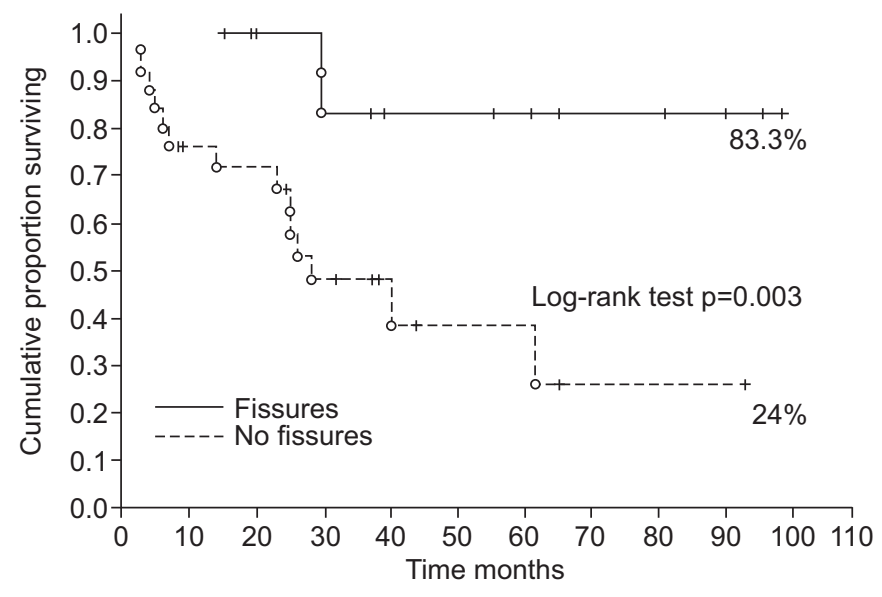

FIGURE 2. Survival comparison between patients with and without visible fissures. O: censored patients; I: alive patients.

improvement in long-term control of symptoms and survival. The airway bypass was investigated [10, 11] and assessed in a multicentre clinical study on 35 patients [21] with homogeneous emphysema. This procedure was designed to facilitate lung deflation and improve expiratory flow and respiratory mechanics; it is achieved by puncturing the wall of segmental bronchi and inserting a dedicated stent to create internal bronchopulmonary communications "bypassing" the "high resistance" airway during expiration. This procedure has been proposed for patients with homogeneous emphysema, in which collateral ventilation allows a preferential route of airflow through the artificial airway with a uniform deflation of the lung. In that study, the airway bypass contributed to reduce hyperinflation and to improve pulmonary function and dyspnoea. One patient died due to massive bleeding during the procedure. The duration of benefit appears to correlate with the degree of pre-treatment hyperinflation and bypass patency. Patients with heterogeneous emphysema are not ideal candidates for this procedure, as collateral ventilation within the whole lung is less pronounced and uniform deflation is more difficult to achieve. For this reason they have been approached using other endobronchial techniques. Some of these procedures should still be considered experimental; however, BLVR with oneway valves have also been extensively tested and validated in clinical trials $[17,22]$. These unidirectional valves allow air to be vented from isolated lung segments or lobes during expiration and prevent air from refilling the parenchyma during inspiration. They functionally isolate the airway supplying the most hyperinflated parts of the lung during inspiration, favouring deflation and even atelectasis. This mechanism should mimic LVRS.

Thus, BLVR with the Zephir ${ }_{\circledR}$ one-way valves represents an attractive procedure. Many reports have previously demonstrated feasibility and safety, with encouraging short- and medium-term results [13-16]. The functional characteristics of the valves have also favoured their use in the case of bullous disease [23], bridge to transplant [24], closure of persistent parenchymal air leaks [25] and overinflation of the contralateral lung after SLT [26]. A multicentre randomised trial on 321 patients with emphysema was published recently [17]. This study was designed to compare safety and efficacy of endobronchial valve therapy in patients with heterogeneous emphysema versus standard medical care; it proposed for BLVR what the NETT trial did for LVRS. The conclusions of that study showed that greater radiographic evidence of heterogeneity (as assessed by HRCT) and fissure completeness were associated with an enhanced response to treatment. However, the results of that trial were somehow questionable, as overall improvement in lung function, exercise tolerance and symptoms were modest; this mild improvement was achieved at the cost of more frequent COPD exacerbations, pneumonia and haemoptysis. These results resemble the discouraging initial interpretation of the LVRS NETT trial. In that case, a more careful analysis was required [20] to draw definitive conclusions and find a specific subgroup of patients likely to benefit. Also, for the BLVR trial, a more careful assessment of the results allows more encouraging conclusions. Only heterogeneity (the difference in emphysema percentage between lobes in the treated lung) remained as an interaction in the multivariate mixed model for both FEV1 and the 6MWT. This was also true in the case of fissure integrity for FEV1, the FEV1/ FVC ratio and the $6 \mathrm{MWT}$. The enhancing effect of heterogeneity (with a cut-off at a median heterogeneity score of $15 \%$ ) sustained the finding of greater FEV1 and 6MWT improvements in the high heterogeneity group $(10.7 \%, p=0.004$ and $12.4 \%, p=0.002$, respectively). Patients with complete fissures showed improvements in FEV1 of $16.2 \%$ and $17.8 \%$ at 6 and 12 months, respectively ( $\mathrm{p}<0.001$ for both), in contrast to insignificant changes of $2 \%$ and $2.8 \%$, respectively, in the group with incomplete fissures; this was indeed the most favourable variable in that study. The development of atelectasis was not specifically investigated in that trial, although previous single-centre reports were able to individuate it as a favourable prognostic variable [27].

Our previous report [13] was in line with the results obtained in the trial and in other single-centre studies [14, 15]. However, outcome needed to be validated with longer follow-up. Only one study reported a long-term extension of a previous pilot study on a small number of patients (16 patients with a follow-up of $>12$ months) [28]. In that study, both unilateral and bilateral BLVR were included; the authors demonstrated that a selected group of patients (six (37.5\%) out of 16) may achieve long-term sustained improvements in pulmonary function; better results were observed in those with higher hyperinflation at baseline and higher TLC and RV/TLC ratio. There were no differences in baseline FEV1, FVC and DL,CO between responders and nonresponders.

The present study, performed on the largest single-centre group of patients available to date, extended the period of follow-up and confirmed our previous short-term encouraging results. FEV1 was also significantly improved at these longer time-points; supplemental oxygen requirements, $6 \mathrm{MWT}$ and MRC were also improved. The percentage of improvement looks higher in patients with an FEV1 below the median, opening a new scenario for patients unsuitable for LVRS. These results were clearly more marked in the group of patients with visible fissures at HRCT. In particular, $50 \%$ of 18 patients assessable at 3 yrs and $72 \%$ of nine at 5 yrs had complete fissures, justifying the stable improvement at these time-points and demonstrating an advantage of this viable during long-term follow-up. Complete fissures prevent interlobar collateral ventilation and guarantee better results, and this explains why patients with complete fissures showed functional and survival advantages at long-term follow-up. This variable will be evaluated more reliably with the new 
technology available nowadays; in fact, an endobronchial catheter is currently under evaluation to assess endobronchial flows from adjacent lobes and resistance of collateral channels. This system relies on the measurement of spontaneous airflow from a sealed and isolated target compartment during spontaneous ventilation in awake subjects. The identification of the critical value of collateral channel resistance above which atelectasis or volume reduction occurs will certainly help to exclude patients with less favourable results [29]. CT with volume rendering may contribute to improving the evaluation of hyperinflation before and after valve placement [30].

Mortality was significant during the study period ( $40 \%$ overall); however, no deaths were related to the procedure. Lung cancer, COPD progression with intractable end-stage respiratory failure and complications after lung transplantation were the main causes of death. We recorded an increased mortality during the follow-up period in patients with nonvisible fissures at HRCT.

Overall, BLVR is confirmed to be feasible and safe. Morbidity is low if the selection of patients is performed carefully. Specific subgroups with marked hyperinflation, clear heterogeneity and the presence of interlobar fissures at HRCT are most likely to benefit from this endoscopic, low-invasive procedure.

\section{STATEMENT OF INTEREST}

None declared.

\section{ACKNOWLEDGEMENTS}

We would like to thank L. Ventura for his support in performing statistical analysis.

\section{REFERENCES}

1 Halbert RJ, Natoli JL, Gano A, et al. Global burden of COPD: systematic review and meta-analysis. Eur Respir J 2006; 29: 523-532.

2 De Giacomo T, Rendina EA, Venuta F, et al. Bullectomy is comparable to lung volume reduction in patients with end stage emphysema. Eur J Cardiothorac Surg 2002; 22: 357-362.

3 Cassivi SD, Meyers BF, Battafarano RJ, et al. Thirteen-year experience in lung transplantation for emphysema. Ann Thorac Surg 2002; 74: 1663-1669.

4 Ciccone AM, Meyers BF, Guthrie TJ, et al. Long term outcome of bilateral lung volume reduction in 250 consecutive patients with emphysema. J Thorac Cardiovasc Surg 2003; 125: 513-525.

5 National Emphysema Treatment Trial Research Group. Patients at high risk of death after lung volume reduction surgery. $N$ Engl J Med 2001; 345: 1075-1083.

6 Watanabe Y, Matsuno K, Tamaoki K, et al. Bronchial occlusion with endoluminal Watanabe spigots. J Bronchol 2003; 10: 264-267.

7 Reilly J, Washko G, Pinto-Plata V, et al. Biological lung volume reduction. A new bronchoscopic therapy for advanced emphysema. Chest 2007; 131: 1108-1113.

8 Hearh FJ, Eberhard RE, Gompelmann D, et al. Bronchoscopic lung volume reduction with a dedicated coil: a clinical pilot study. Ther Adv Respir Dis 2010; 4141: 225-231.

9 Emery MJ, Eveland RL, Eveland K, et al. Lung volume reduction by bronchoscopic administration of steam. Am J Respir Crit Care Med 2010; 182: 1282-1291.

10 Rendina EA, De Giacomo T, Venuta F, et al. Feasibility and safety of the air way by-pass procedure for patients with emphysema. J Thorac Cardiovasc Surg 2003; 125: 1294-1299.
11 Choong CK, Phen L, Massetti P, et al. Prolongation of patency of airway bypass stents with use of drug-eluting stents. J Thorac Cardiovasc Surg 2006; 131: 60-64.

12 Fann JI, Berry GJ, Burden TA. Bronchoscopic approach to lung volume reduction using a valve device. J Bronchol 2003; 10: 253-259.

13 Venuta F, De Giacomo T, Rendina EA, et al. Bronchoscopic lung volume reduction with one way valves in patients with heterogeneous emphysema. Ann Thorac Surg 2005; 79: 411-416.

14 Toma TP, Hopkinson NS, Hiller J, et al. Bronchoscopic volume reduction with valve implants in patients with severe emphysema. Lancet 2003; 361: 931-933.

15 Snell GI, Helsworth L, Borrill ZL, et al. The potential for bronchoscopic lung volume reduction using bronchial prosthesis: a pilot study. Chest 2003; 124: 1073-1080.

16 Yim AP, Hwong TM, Lee TW, et al. Early results of endoscopic lung volume reduction for emphysema. J Thorac Cardiovasc Surg 2004; 127: 1564-1573.

17 Sciurba FC, Ernst A, Herth FJF, et al. A randomized study of endobronchial valves for advanced emphysema. $N$ Engl J Med 2010; 363: 1233-1244.

18 Venuta F, Rendina EA, De Giacomo T, et al. Bronchoscopic procedures for emphysema treatment. Eur J Cardiothorac Surg 2006; 29: 281-287.

19 Fishman A, Martinez F, Naunheim K, et al. A randomized trial comparing lung volume reduction surgery with medical therapy for severe emphysema. N Engl J Med 2003; 348: 2059-2073.

20 Sanchez PG, KuCharczuk JC, Su S, et al. National Emphysema Treatment Trial redux: accentuating the positive. J Thorac Cardiovasc Surg 2010; 140: 564-572.

21 Cardoso PF, Snell GI, Hopkins P, et al. Clinical application of airway bypass with paclitaxel-eluting stents: early results. J Thorac Cardiovasc Surg 2007; 134: 974-981.

22 Wood DE, McKenna RJ, Yusem RD, et al. A multicenter trial of an intrabronchial valve for treatment of severe emphysema. J Thorac Cardiovasc Surg 2007; 133: 65-73.

23 Santini M, Fiorello A, Di Crescenzo VG, et al. Use of unidirectional valves for the treatment of giant emphysematous bulla. J Thorac Cardiovasc Surg 2010; 139: 224-226.

24 Venuta F, Diso D, Anile M, et al. Bronchoscopic lung volume reduction as a bridge to lung transplantation in patients with chronic obstructive pulmonary disease. Eur J Cardiothorac Surg 2011; 39: 364-367.

25 Travaline JM, McKenna RJ Jr, De Giacomo T, et al. Treatment of persistent pulmonary air leaks using endobronchial valves. Chest 2009; 136: 355-360.

26 Pato O, Rama P, Allegne M, et al. Bronchoscopic lung volume reduction in a single lung transplant recipient with natal lung hyperinflation: a case report. Transplant Proc 2010; 42: 1979-1981.

27 Hopkinson NS, Kemp SW, Toma TP, et al. Atelectasis and survival after bronchoscopic lung volume reduction for COPD. Eur Respir J 2011; 37: 1346-1351.

28 Kotecha S, Westall G, Holsworth L, et al. Long-term outcomes from bronchoscopic lung volume reduction using a bronchial prosthesis. Respirology 2011; 16: 167-173.

29 Aljuri N, Freitag L. Validation and pilot clinical study of a new bronchoscopic method to measure collateral ventilation before endobronchial lung volume reduction. J Appl Physiol 2009; 106: 774-783.

30 D'Andrilli A, Vismara L, Rolla M, et al. Computed tomography with volume rendering for the evaluation of parenchymal hyperinflation after bronchoscopic lung volume reduction. Eur J Cardiothorac Surg 2009; 35: 403-407. 